\title{
Comparação dos parâmetros de qualidade de comprimidos de atenolol similares e genéricos em relação ao medicamento referência
}

\author{
Quality parameters comparison between similar and generic atenolol \\ tablets and the reference product
}

Recebido em: 06/09/2019 Aceito em: $17 / 08 / 2020$
Emilly Isabelli dos Santos TEODORO ${ }^{1}$; Thalita Prates da SILVA ${ }^{1}$; Mariana Nascimento de PAULA ${ }^{1}$; João Carlos Palazzo de MELLO' Mariana Aparecida LOPES-ORTIZ²; Daniela Cristina de Medeiros ARAÚJO² ${ }^{1}$ Universidade Estadual de Maringá. Av. Colombo, 5790, Zona 7. Maringá, PR, Brasil. ${ }^{2}$ Centro Universitário Ingá-Uningá. Rod. PR 317, 6114, Parque Industrial. Maringá, PR. E-mail:danielamedeiros@hotmail.com

\section{ABSTRACT}

Pharmaceutical equivalence between two medicinal products corresponds to the proof that both contain the same drug, i.e., the same active ingredient or salt, the same concentration, pharmaceutical form and route of administration, containing or not identical excipients. Tests conducted on comparative quality analysis include physico-chemical tests and when applicable, microbiological, and biological tests. This study evaluated the quality parameters of two brands of generic (called D and E) and two brands of similar (B and C) were evaluated in relation to the reference drug (A) of atenolol $50 \mathrm{mg}$. Tests of weight variation, friability, disintegration and assay were performed. In addition, microbiological control of the samples was performed by counting the total number of mesophilic microorganisms. The result was that there was no growth of colonies. All samples tested met the requirements of the tests performed according to the parameters described in the Brazilian Pharmacopeia 6th edition.

Keywords: comparative study; atenolol; generic drugs; similar drugs.

\section{RESUMO}

A equivalência farmacêutica entre dois medicamentos corresponde à comprovação de que ambos contêm o mesmo fármaco; isso é; mesmo sal ou éster da mesma molécula terapeuticamente ativa; mesma concentração; forma farmacêutica e via de administração; podendo ou não conter excipientes idênticos. Os testes realizados para análise comparativa de qualidade incluem ensaios físico-químicos e; quando aplicáveis; microbiológicos e biológicos. Neste trabalho foram avaliados os parâmetros de qualidade de duas marcas de medicamento genérico (denominadas D e E) e duas marcas de similar (B e C) em relação ao medicamento referência (A) de atenolol $50 \mathrm{mg}$. Foram realizados os testes de peso médio; friabilidade; desintegração e doseamento. Além disso; o controle microbiológico das amostras foi realizado por meio da contagem do 
número total de micro-organismos mesofílicos. O resultado foi que não ocorreu crescimento de colônias. Todas as amostras testadas cumpriram os requisitos dos testes realizados; de acordo com os parâmetros descritos na Farmacopeia Brasileira $6^{\mathrm{a}}$ edição.

Palavras-chave: estudo comparativo; atenolol; medicamento genérico; medicamento similar.

\section{INTRODUÇÃO}

O mercado farmacêutico vem constantemente evoluindo com a demanda da saúde, inovando tecnologicamente e modificando os sistemas de saúde. Boa parte dessa mudança que ocorre na indústria se dá pela transformação demográfica e epidemiológica do meio, para atender a necessidade do país pela demanda de saúde, e consequentemente mudando o cenário econômico (1).

Muito se discute sobre a qualidade, segurança e eficácia dos medicamentos genéricos e similares comercializados no Brasil. Estudos de equivalência farmacêutica e perfil de dissolução de medicamentos genéricos e similares, comercializados no mercado nacional e internacional, têm demonstrado diferenças significativas entre as preparações, alertando para a falta da intercambialidade (2).

A equivalência farmacêutica entre dois medicamentos corresponde à comprovação de que ambos contêm o mesmo fármaco, isso é, mesmo sal ou éster da mesma molécula terapeuticamente ativa, mesma concentração, forma farmacêutica e via de administração, podendo ou não conter excipientes idênticos (3). A Resolução da Diretoria Colegiada (RDC) 31/2010, da Agência Nacional de Vigilância Sanitária (Anvisa), dispõe sobre os requisitos para a realização dos estudos de Equivalência Farmacêutica e de perfil de dissolução comparativo (4). Os estudos de Equivalência Farmacêutica destinam-se à avaliação da qualidade dos medicamentos por meio de análise comparativa entre o medicamento teste e o medicamento de referência. Os testes realizados incluem ensaios físico-químicos e, quando aplicáveis, microbiológicos e biológicos que constam na monografia do medicamento na Farmacopeia Brasileira (3), ou em outro compêndio oficial (5).

$O$ atenolol pertence à classe dos $\beta$-bloqueadores adrenérgicos utilizados em cardiopatia is- quêmica, insuficiência cardíaca e principalmente como anti-hipertensivo. Esse medicamento atua em receptor $\beta$-1-adrenérgico, como um antagonista seletivo, promovendo vasodilatação e redução da pressão arterial. O atenolol pode ser associado a um diurético, principalmente em pacientes idosos (6).

Neste trabalho foram analisadas duas marcas de medicamento similar e duas marcas de medicamento genérico, em comparação ao respectivo medicamento referência. $\mathrm{O}$ objetivo foi comparar os parâmetros de qualidade dos medicamentos genéricos e similares em relação ao medicamento referência.

\section{MATERIAL E MÉTODO}

Os medicamentos contendo atenolol $50 \mathrm{mg}$ foram adquiridos em farmácias comerciais e atenolol grau farmacêutico (Galena 99,5\%, L. ALA/ P1604029) em uma farmácia de manipulação na cidade de Maringá, PR, no período de junho de 2018.

Os comprimidos de atenolol $50 \mathrm{mg}$ foram identificados como amostra A (referência); amostra B e C (similar); e amostra D e E (genérico). Os testes realizados foram determinação de peso, friabilidade, desintegração, contagem do número total de microrganismos mesofílicos, conforme descrito na Farmacopeia Brasileira $6^{\mathrm{a}}$ edição (3), e o doseamento foi uma adaptação da Farmacopeia Brasileira (3).

Determinação de peso. Foram pesados 20 comprimidos de cada produto em balança semi-analítica (Celtac modelo FA2104N), seguida da determinação da variação percentual do peso dos comprimidos em relação à média.

Friabilidade. Para realização deste teste foram pesados um total de 20 comprimidos de cada amostra, tendo em vista que o peso médio dos comprimidos foi inferior a $0,65 \mathrm{~g}$. Após a pesagem, os 
comprimidos foram colocados no friabilômetro (Nova Ética modelo 300), e submetidos à ação do aparelho por 4 minutos a 25 rotações/minuto. Após o teste, os comprimidos íntegros e livres de pó foram recolhidos e novamente pesados para calcular a porcentagem de friabilidade.

Desintegração. O teste de desintegração foi realizado com 6 comprimidos de cada amostra, utilizando água destilada a $37 \pm 1{ }^{\circ} \mathrm{C}$ como líquido de imersão em equipamento desintegrador (Nova Ética modelo 301-AC). Após 30 minutos, foi verificado se os comprimidos se desintegraram completamente ou não.

Doseamento. Para determinar a quantidade de substância ativa em cada amostra, foram utilizados 20 comprimidos pulverizados.

Preparo da solução de atenolol: 0,05 g de atenolol foram dissolvidos em $50 \mathrm{~mL}$ de metanol, e a mistura foi aquecida em banho maria a $60{ }^{\circ} \mathrm{C}$ por 10 minutos, com posterior homogeneização em agitador magnético (Fisatom modelo 752A) por 15 minutos. Após o tempo decorrido, foram realizadas diluições seriadas até a concentração de $0,01 \%(\mathrm{p} / \mathrm{v})$.

Preparo das amostras: uma quantidade de pó equivalente a $0,05 \mathrm{~g}$ de atenolol de cada amostra foi pesada em triplicata e foram adicionados $50 \mathrm{~mL}$ de metanol. A mistura foi aquecida em banho maria a $60{ }^{\circ} \mathrm{C}$ por 10 minutos, com posterior homogeneização em agitador magnético por 15 minutos. Após a homogeneização, a mistura foi transferida para balão de $50 \mathrm{~mL}$, o volume foi completado com o mesmo solvente e então filtrado. Foram realizadas diluições seriadas até a concentração de $0,01 \%(\mathrm{p} / \mathrm{v})$.

Após preparo das amostras, foi realizada a leitura em espectrofotômetro de absorção no ultravioleta (Ocean Optics modelo UBS 2000+) em comprimento de onda de $275 \mathrm{~nm}$ e comparado com a solução de atenolol (substância grau farmacêutico - Galena 99,5\%). Foi utilizado metanol para ajuste do zero e a quantidade de atenolol nos comprimidos foi calculada a partir das leituras obtidas.

Contagem do número total de micro-organismos mesofílicos. $\mathrm{O}$ teste foi realizado em duplicata, empregando-se Bico de Bunsen para manter ambiente estéril. Foram utilizados o meio ágar caseína soja (TSA) para analisar o crescimento de microrganismos aeróbios totais e o meio ágar Sabouraud dextrose para avaliar crescimento de fungos e leveduras.

Preparo das amostras: o comprimido foi colocado em tubos preenchidos com $5 \mathrm{ml}$ de caldo caseína soja e homogeneizados em vortex até a completa desintegração.

Preparo das placas: foi pipetado $1 \mathrm{ml} \mathrm{da}$ amostra em uma placa de petri e em seguida adicionado TSA ou ágar Sabouraud dextrose. A amostra foi homogeneizada na placa segundo a técnica de Pour Plate. Depois do ágar solidificado, as placas foram mantidas em temperatura controlada de 32,5 ${ }^{\circ} \mathrm{C} \pm 2,5{ }^{\circ} \mathrm{C}$ durante 5 dias para as amostras com ágar caseína soja e $22,5{ }^{\circ} \mathrm{C} \pm 2,5^{\circ} \mathrm{C}$ durante 7 dias para o ágar Sabouraud dextrose.

Ao final do teste foi observada a ocorrência ou não de crescimento de bactérias e fungos nos meios de cultura.

\section{RESULTADOS E DISCUSSÃO}

Teste de determinação de peso. $O$ peso do comprimido está relacionado à quantidade de princípio ativo e excipientes utilizados na formulação (7). A regulação das máquinas de compressão é outro fator relevante nesse processo, podendo ocorrer variações de peso em um mesmo lote de comprimidos (8).

Os valores obtidos na determinação de peso dos comprimidos de atenolol $50 \mathrm{mg}$ (A, B, C, D e E) estão descritos na Tabela 1.

Segundo parâmetros estabelecidos pela Farmacopeia Brasileira para comprimidos com peso médio maior que $80 \mathrm{mg}$ e menor que $250 \mathrm{mg}$ o limite de variação permitido é de $\pm 7,5 \%$, podendo ter apenas duas unidades fora desse limite, mas nenhuma pode estar acima ou abaixo do dobro da porcentagem indicada (9). Sendo assim, todas as amostras testadas foram aprovadas neste parâmetro de qualidade.

Em um estudo de equivalência farmacêutica e perfil de dissolução comparativo de medicamentos contendo hidroclorotiazida, os valores de peso 
Tabela 1. Determinação da variação de peso de comprimidos de atenolol $50 \mathrm{mg}$.

\begin{tabular}{|l|c|c|c|c|c|}
\hline & Amostra A & Amostra B & Amostra C & Amostra D & Amostra E \\
\hline Maior peso (g) & 0,2094 & 0,2210 & 0,2115 & 0,2217 & 0,1537 \\
\hline Menor peso (g) & 0,2045 & 0,2014 & 0,2033 & 0,2038 & 0,1421 \\
\hline Peso Médio (g) & $\mathbf{0 , 2 0 7 3}$ & $\mathbf{0 , 2 1 2 1}$ & $\mathbf{0 , 2 0 6 2}$ & $\mathbf{0 , 2 1 3 2}$ & $\mathbf{0 , 1 4 9 0}$ \\
\hline Mais 7,5\% (g) & 0,2229 & 0,2280 & 0,2217 & 0,2292 & 0,1601 \\
\hline Menos 7,5\% (g) & 0,1918 & 0,1962 & 0,1907 & 0,1972 & 0,1378 \\
\hline DP & 0,0013 & 0,0044 & 0,0023 & 0,0040 & 0,0023 \\
\hline CV (\%) & 0,64 & 2,08 & 1,13 & 1,92 & 1,58 \\
\hline Resultado & Aprovado & Aprovado & Aprovado & Aprovado & Aprovado \\
\hline
\end{tabular}

médio mostram essa relação com a quantidade de ativo e excipiente, sendo os comprimidos mais pesados os que possuem uma quantidade maior de excipiente na sua formulação. No estudo, o medicamento referência apresentou os valores mais baixos e a amostra $\mathrm{G} 3$ valores maiores, indicando que essa amostra possui mais excipientes do que os demais comprimidos (10).

Teste de friabilidade. Este teste permite avaliar a resistência dos comprimidos a abrasão quando submetidos a ação mecânica semelhante a sofrida durante os processos de transporte, manuseio, emblistagem, etc. (7). Para que ocorra a aprovação no teste é preciso que os valores de perda de massa dos comprimidos após o teste sejam inferiores a 1,5\% (9). Os comprimidos também não devem apresentar rachadura, lascas ou algum outro dano visível após o término do teste (2). Os resultados obtidos no teste de friabilidade estão descritos na Tabela 2.

Os dados fornecidos na Tabela 2 indicam que todas as amostras foram aprovadas no teste de friabilidade, não sendo apresentado perda de massa su- perior a 1,5\%, conforme critério descrito na Farmacopeia Brasileira (9). Foi observado também que a porcentagem de perda no teste não ultrapassou $0,6 \%$ nas amostras analisadas.

Resultado semelhante foi obtido no estudo de Buzzi e cols. (2006) que realizaram uma avaliação da qualidade de comprimidos de atenolol genérico e similar (11). No teste de friabilidade, as amostras testadas não apresentaram perda maior que $0,4 \%$.

Em um estudo de equivalência farmacêutica e perfil de dissolução de medicamentos genéricos contendo paracetamol, obteve-se uma perda máxima de $0,39 \%$ no teste de friabilidade, sem presença de rachaduras ou lascas nos comprimidos ao final do teste (2).

Em um estudo de equivalência farmacêutica realizado com comprimidos de cloridrato de bupropiona em medicamentos similares, das 11 amostras analisadas 3 apresentaram valor de friabilidade maior que o obtido pelo medicamento referencia $(0,3 \%)$, no entanto, todas as amostras foram aprovadas com valores inferiores a $1,5 \%(12)$.

Tabela 2. Resultados obtidos no teste de friabilidade dos comprimidos de atenolol $50 \mathrm{mg}$.

\begin{tabular}{|l|c|c|c|c|c|}
\hline & Amostra A & Amostra B & Amostra C & Amostra D & Amostra E \\
\hline Peso inicial (g) & 4,150 & 4,314 & 4,140 & 4,232 & 2,988 \\
\hline Peso final (g) & 4,134 & 4,290 & 4,122 & 4,210 & 2,986 \\
\hline Perda (\%) & 0,387 & 0,559 & 0,436 & 0,522 & 0,066 \\
\hline Resultado & Aprovado & Aprovado & Aprovado & Aprovado & Aprovado \\
\hline
\end{tabular}


Teste de desintegração. A desintegração é uma etapa crucial para a dissolução e posterior absorção do fármaco. O teste de desintegração é realizado utilizando um desintegrador, em condições experimentais específicas (meio de imersão, tem- po e temperatura). Ao final do teste é observado se ocorreu a desintegração total dos comprimidos ou a presença de massa sem núcleo palpável (7).

Os resultados do teste de desintegração podem ser observados no Quadro 1.

Quadro 1. Resultados obtidos no teste de desintegração dos comprimidos de atenolol $50 \mathrm{mg}$.

\begin{tabular}{|c|c|c|c|c|}
\hline \multicolumn{1}{|c|}{ Amostra A } & \multicolumn{1}{|c|}{ Amostra B } & Amostra C & \multicolumn{1}{c|}{ Amostra D } & Amostra E \\
\hline $\begin{array}{l}\text { Todos os } 6 \\
\text { comprimidos } \\
\text { desintegrados em } \\
30 \text { minutos. }\end{array}$ & $\begin{array}{l}\text { Todos os } 6 \\
\text { comprimidos } \\
\text { desintegrados em } \\
\text { 30 minutos. }\end{array}$ & $\begin{array}{l}\text { Todos os } 6 \text { comprimidos } \\
\text { desintegrados em } \\
\text { 30 minutos. Apresentou } \\
\text { resíduos insolúveis, mas } \\
\text { sem núcleo palpável. }\end{array}$ & $\begin{array}{l}\text { Todos os } 6 \\
\text { comprimidos } \\
\text { desintegrados em }\end{array}$ & $\begin{array}{l}\text { Todos os } 6 \\
\text { comprimidos } \\
\text { desintegrados em } \\
\text { Aprovado }\end{array}$ \\
\hline Aprovado & Aprovado & Aprovado & Aprovado \\
\hline
\end{tabular}

De acordo com a Farmacopeia Brasileira (9), o tempo máximo permitido para desintegração de comprimidos de atenolol é 30 minutos. Todos os comprimidos testados desintegraram antes do tempo limite, sendo aprovados neste teste.

Em um estudo semelhante de avaliação de comprimidos de atenolol, os tempos de desintegração dos comprimidos não chegaram a $3 \mathrm{mi}$ nutos (13). Em teste de desintegração realizado com comprimidos de paracetamol foi possível observar que as amostras se desintegraram em 6 minutos (2).

Doseamento. De acordo com a monografia do atenolol comprimidos descrita na Farmacopeia Brasileira (9), o teor de atenolol em comprimidos pode variar de 90 a $110 \%$ do valor declarado no rótulo. Os resultados de teor obtidos para cada amostra estão descritos na Tabela 3 .

Tabela 3. Valores obtidos no doseamento dos comprimidos de atenolol $50 \mathrm{mg}$.

\begin{tabular}{|l|c|c|c|c|c|}
\hline & Amostra A & Amostra B & Amostra C & Amostra D & Amostra E \\
\hline Teor 1 (\%) & 103,40 & 99,40 & 90,74 & 91,00 & 97,40 \\
\hline Teor 2 (\%) & 106,60 & 96,80 & 100,20 & 97,00 & 97,40 \\
\hline Teor 3 (\%) & 99,80 & 105,80 & 98,00 & 93,00 & 109,40 \\
\hline Média (\%) & 103,26 & 100,66 & 96,31 & 93,66 & 101,40 \\
\hline DP (\%) & 0,034 & 0,046 & 0,049 & 0,030 & 0,069 \\
\hline CV (\%) & 0,032 & 0,046 & 0,051 & 0,032 & 0,068 \\
\hline
\end{tabular}

Todas as amostras analisadas foram aprovadas por estarem dentro do limite aceitável de 90 a $110 \%$ de substância ativa. No entanto, por ser uma adaptação da monografia, se faz necessário a realização de teste futuros para a comprovação dos resultados.

Em um estudo de medicamentos genéricos contendo atenolol $100 \mathrm{mg}$, os comprimidos testa- 
dos apresentaram um teor entre $99 \%$ e $102,5 \%$ do valor declarado (13). Na avaliação da qualidade de comprimidos de atenolol $50 \mathrm{mg}$ genéricos e similares as amostras testadas apresentaram teor entre $91 \%$ a $110 \%$ do valor declarado, sendo que a amostra B apresentou valor correspondente ao limite superior aceitável (11).

Em outro estudo comparativo de métodos de análise de comprimidos de atenolol utilizando espectroscopia ultravioleta e titulação potenciométrica, foram obtidos resultados de teor entre $90 \%$ a $110 \%$, estando as amostras aprovadas neste parâmetro (14).

Teste de contagem do número total de micro-organismos mesofílicos. De acordo com a Farmacopeia Brasileira (3) são registradas, como resultado, apenas placas que tenham crescimento inferior a 250 colônias para bactérias e, para fungos, abaixo de 50 colônias.

Segundo critérios estabelecidos na Farmacopeia Brasileira (3) os limites máximos aceitáveis de crescimento microbiano em comprimidos são de $10^{3} \mathrm{UFC} / \mathrm{mL}$ para a contagem das bactérias, e de $10^{2} \mathrm{UFC} / \mathrm{mL}$ para a contagem de fungos.

Nesse teste não foi observado o crescimento de bactérias e fungos nas amostras analisadas; estando todas aprovadas segundo critérios estabelecidos pela Farmacopeia Brasileira $6^{\mathrm{a}}$ edição (3).

Mesmo com o advento da lei dos genéricos (15) que afirma que estes medicamentos devem ter comprovada eficácia, segurança e qualidade e são intercambiáveis com o medicamento referência e da RDC 58/2014, que determina que todos os medicamentos similares obrigatoriamente devem apresentar testes de bioequivalência com o medicamento referência (16), alguns prescritores e parte da população ainda têm resistência ao uso de medicamentos similares e genéricos, provavelmente devido à falta de informação sobre o tema.

Em uma pesquisa sobre aceitação de medicamentos genéricos por pacientes com doenças crônicas, foi realizada uma intervenção educativa enfatizando as vantagens do genérico, como sua bioequivalência terapêutica, qualidade e baixo custo e, sugerindo a intercambialidade dos medicamentos prescritos por genéricos. A pesquisa mos- trou que a maioria dos pacientes aceitaram a mudança, e poucos voltaram ao seu tratamento antigo, independente do grau de escolaridade do paciente, após as informações e orientações prestadas pelos prescritores sobre o medicamento genérico (17).

Em um estudo realizado com 273 pessoas sobre a importância da intercambialidade dos medicamentos genéricos e similares, mostrou que 136 não sabiam o que era medicamento similar equivalente, 15 não sabiam o que era medicamento genérico e 123 não sabiam o que era medicamento referência; estando relacionado ao grau de escolaridade dos indivíduos também. Quando questionados se acreditam que os medicamentos genéricos têm o mesmo efeito que o referência, apenas 70 entrevistados não acreditam nesse efeito, porém 99 desconfiam do genérico devido ao seu preço mais baixo; e 225 pessoas ainda não possuem conhecimento sobre alguns testes de qualidade, como a equivalência e bioequivalência, ou seja, ainda é necessário que mais informações sobre os genéricos e similares sejam passadas a população, sendo por meio de estudos ou orientações profissionais (18).

Outra pesquisa sobre medicamentos genéricos, feita em um município de Rondônia com 357 indivíduos, apresentou resultados relacionados a escolaridade, utilização, resultado esperado e influência do preço dos genéricos, entre outras questões. O estudo mostrou que $76 \%$ das pessoas já fizeram uso de medicamentos genéricos, e 23\% não obtiveram o resultado esperado, no entanto fatores como a falta de adesão ao tratamento podem estar relacionados a esse resultado, e não necessariamente a falta de qualidade do medicamento. Entre os entrevistados, $75 \%$ responderam que o valor do medicamento influencia no momento da compra, sendo um dos principais fatores para a aquisição e uso dos medicamentos genéricos nesse estudo. Apesar da aceitação, ainda devem ser feitos mais estudos para informar a população sobre a segurança dos medicamentos genéricos e similares (19).

Desta forma, justifica-se a realização de estudos de avaliação de qualidade, equivalência farmacêutica e bioequivalência de genéricos e similares, para comprovar a segurança e eficácia destes medicamentos. 


\section{CONCLUSÃO}

De acordo com os testes realizados pode ser concluído que os medicamentos testados (genéricos e similares) demonstraram estar adequados na avaliação da qualidade, em relação ao medicamento referência quanto aos testes de variação de peso, friabilidade, desintegração e doseamento. As amostras também apresentaram resultados semelhantes aos obtidos pelo medicamento referência quanto à contagem do número total de micro-organismos mesofílicos. Os resultados obtidos sugerem que os medicamentos testados estejam em conformidade com os parâmetros farmacopeicos, com relação aos testes realizados, segundo os limites preconizados pelo Farmacopeia Brasileira $6^{\mathrm{a}}$ edição.

\section{AGRADECIMENTOS}

Os autores agradecem ao Laboratório Palafito da Universidade Estadual de Maringá (UEM) e também ao Centro Universitário Ingá (UNINGÁ).

\section{REFERÊNCIAS}

1. Reis C; Pimentel V; Pieroni JP \& Mitidieri T. Panoramas setoriais 2030: indústria farmacêutica. In: Panoramas setoriais 2030: desafios e oportunidades para o Brasil. Rio de Janeiro: Banco Nacional de Desenvolvimento Econômico e Social. 2017. p. 137-146.

2 Brum TF; Laporta LV; Pons RPJ; Gonçalves CA; Santos MR. Equivalência farmacêutica e estudo comparativo dos perfis de dissolução de medicamentos genéricos contendo paracetamol. Rev Ciênc Farm Básica Apl. 2012;33(3):373-378.

3 BRASIL. Farmacopeia Brasileira 6 ed. Agência Nacional de Vigilância Sanitária. Brasília: ANVISA, 2019. v. 1

4 BRASIL. Resolução de Diretoria Colegiada (RDC) $\mathrm{N}^{\mathrm{o}}$ 31, de 11 de agosto de 2010. Dispõe sobre a realização dos Estudos de Equivalência Farmacêutica e de Perfil de Dissolução Comparativo. Ministério da Saúde. Agência Nacional de Vigilância Sanitária. Diário Oficial da União, Brasília, DF, 12 ago. 2010.

5 BRASIL. Resolução de Diretoria Colegiada (RDC) No 37 , de 6 de julho de 2009. Dispõe sobre da admissibilidade das Farmacopéias estrangeiras. Ministério da Saúde. Agência Nacional de Vigilância Sanitária. Diário Oficial da União, Brasília, DF.

6 Martelli A; Longo MAT; Seriani C. Aspectos clínicos e mecanismo de ação das principais classes farmacológicas usadas no tratamento da hipertensão arterial sistêmica. Estud Biol. 2008;30(70/71/72):149-156.

7 Allen JR LV; Popovich NG \& Ansel HC. Formas Farmacêutica e Sitemas de Liberação de Fármacos. 9th. ed. São Paulo: Artmed. 2013.

8 Prista LN; Alves AC; Morgado R; Lobo JS. Tecnologia farmacêutica. 6th. ed. Lisboa: Fundação Calouste Gunlbenkian.2003.

9 BRASIL. Farmacopeia Brasileira 6 ed. Agência Nacional de Vigilância Sanitária. Brasília: ANVISA, 2019. v. 2.
10 Ribeiro CC; Andrade GMM; Couto RO. Estudos de equivalência farmacêutica e perfil de dissolução comparativo de medicamentos contendo Hidroclorotiazida. Infarma - Cienc Farmac. 2018;30(1):5-13. DOI: 10.14450/2318-9312.v30.e1.a2018.pp5-13

11 Buzzi V; Rosseto K; Zétola M; Pezzini B R; Bazzo GC. Avaliação da qualidade de comprimidos de atenolol: estudo comparativo entre medicamentos de referência, genérico e similar. Arq. Ciênc. Saúde Unipar. 2006;10(3):119-122. DOI: 10.25110/arqsaude. v10i3.2006.2267

12 Castro VL. Estudos de Equivalência Farmacêutica com comprimidos do cloridrato de bupropiona em medicamentos similar e de referência. [Dissertação] Goiânia.: Universidade Católica de Góias, PUC-Goiás. 2010 .

13 Weich A; Rolim CMB; Oliveira DC; Goebel K. Validation of UV Spectrophotometric and HPLC Methods for Quantitative Determination of Atenolol in Pharmaceutical Preparations. Lat. Am. J. Pharm. 2007; 26 (5):765-770.

14 Weber TM. Estudo e comparação de métodos de análise de comprimidos de atenolol utilizando a espectroscopia ultravioleta e titulação potenciométrica. [Trabalho de Conclusão de Curso] Porto Alegre: Universidade Federal do Rio Grande do Sul, UFRGS. 2011.

15 BRASIL. Lei No 9.787, de 10 de fevereiro de 1999. Altera a Lei $n^{\circ} 6.360$, de 23 de setembro de 1976, que dispõe sobre a vigilância sanitária, estabelece o medicamento genérico, dispõe sobre a utilização de nomes genéricos em produtos farmacêuticos e dá outras providências. Ministério da Saúde. Agência Nacional de Vigilância Sanitária. Diário Oficial da República Federativa do Brasil, Poder Executivo, Brasília, DF, 10 fev. 1999. 


\section{lonfarma}

16 BRASIL. Resolução de Diretoria Colegiada (RDC) $\mathrm{N}^{\mathrm{o}}$ 58, de 10 de outubro de 2014. Dispõe sobre as medidas a serem adotadas junto à Anvisa pelos titulares de registro de medicamentos para a intercambialidade de medicamentos similares com o medicamento de referência. Ministério da Saúde. Agência Nacional de Vigilância Sanitária. Diário Oficial da União, Brasília, DF.

17 Vallès JA; Barreiro M; Cereza G; Ferro JJ; Martínez MJ; Cucurull E; Barceló E. Aceptación de los fármacos genéricos en equipos de atención primaria: efecto de una intervención educativa y de los precios de referência. Gac Sanit. 2002;16(6):505-510. DOI: 10.1016/S02139111(02)71972-9.
18 Silva AKO; Silva LA; Almeida PS; Khouri AG; Santos SO; Paiva CCS; Silveira AA; Souza APS. A importância da intercambialidade e dos medicamentos genéricos e similares. Rev. Ref. Saúde - FESGO. 2019;2(3):08-21.

19 Silva CM; Valiatti TB; Calazans RSP; Barcelos IB; Salvi JO. Medicamentos genéricos: Uma abordagem no município de Vale do Paraíso, Rondônia. Acta Biomed. Bras. 2017;8(2):83-90. DOI: 10.18571/acbm.142. 